\title{
STYK1/NOK-A Potential Radiotherapeutic Target and Biomarker for Gastric Cancer and Cervical Cancer
}

\author{
Ling Gao ${ }^{1}$, Xiaohua Chen ${ }^{2}$, Zenghai $\mathrm{Ma}^{3}$, Fengsheng $\mathrm{Li}^{4^{*}}$ \\ ${ }^{1}$ Key Laboratory of Radiological Protection and Nuclear Emergency, National Institute for Radiological Protection, China Centers \\ for Disease Control, Beijing, China; ${ }^{2}$ Department of Experimental Therapy of Acute Radiation Sickness, Beijing Institute of Radiation \\ Medicine, Beijing, China; ${ }^{3}$ College of Life Science and Technology, Xinjiang University, Urumchi, China; ${ }^{4}$ The Second Artillery \\ Journal Hospital People's Liberation Army, Beijing, China; \\ Email: *lifs0624@163.com
}

Received November $30^{\text {th }}$, 2012; revised December 31 $1^{\text {st }}$, 2012; accepted January $8^{\text {th }}, 2013$

Copyright (C) 2013 Fengsheng Li et al. This is an open access article distributed under the Creative Commons Attribution License, which permits unrestricted use, distribution, and reproduction in any medium, provided the original work is properly cited.

\begin{abstract}
This article introduced the $S T Y K 1 / N O K$, including its origin, chemical composition and biological function, and the expression of $S T Y K 1 / N O K$ in various cancer cell lines was reviewed. Furthermore, our recent study showed that $S T Y K 1 / N O K$ protein was also over expressed in gastric cancer and cervical cancer specimens, and STYK1/NOK expression increased after tumor cells were irradiated with $\gamma$ ray. These results indicated that $S T Y K 1 / N O K$ might be involved in the occurrence and progress of gastric cancer and cervical cancer, and contribute to the radioresistance of tumor cells. Thus, STYK1/NOK might be a potential therapeutic target and diagnostic marker for gastric cancer and cervical cancer.
\end{abstract}

Keywords: Gastric Cancer; Cervical Cancer; STYK1/NOK; Therapeutic Target

\section{Introduction}

It is well known that receptor tyrosine kinases (RTKs) is perhaps the most important and extensively studied pathway. Ligand binding results in receptor autophosphorylation and the subsequent activation of downstream signalling cascades that include mitogen-activated protein kinase (MAPK), extracellular-signal-regulated kinase (ERK), phosphatidylinositol 3-kinase (PI3K), phospholipase $\mathrm{C} \gamma$, protein kinase $\mathrm{C}$ and small GTPases such as Ras, Rho and RAC1 (Ras-related C3 botulinum toxin substrate 1). Abnormal expression of RTKs was believed to contribute to the tumorigenesis and progression.

STYK1 (Serine/Threonine/Tyrosine Kinase 1), also named NOK (The novel oncogene with kinase-domain) encoding a potential kinase, has been cloned in 2004 by Liu, L. et al. [1]. STYK1/NOK is approximately $30 \%$ similar to the mouse fibroblast growth factor (FGF) and plateletderived growth factor (PDGF) receptor super-family, and is predicted to have a transmembrane domain and protein kinase domain, belonging to a receptor protein tyrosine kinase family. Many studies suggested that $S T Y K 1 / N O K$ were involved in oncogenesis and metastasis [1,2], previous studies have shown that $S T Y K 1 / N O K$ mRNA was

\footnotetext{
*Corresponding author.
}

up-regulated in various cancer, including breast cancer and lung cancer $[3,4]$.

\section{STYK1/NOK Genes Studies Development in Gastric Cancer and Cervical Cancer}

Gastric cancer was the fourth most common cancer and the second leading cause of cancer death worldwide [5-8]. It has been well proved that RTKs are involved in the occurrence and progress of gastric cancer. Furthermore, RTKs-targeted agents, such as Cetuximab [9], Trastuzumab [10-12], have been applied for treatment of the gastric cancer.

Besides melanoma cells (B16), lung cancer cells (A549), breast cancer cells (MCF-7, MDA-MD-123), the expression of $S T Y K 1 / N O K$ was detected in gastric cancer cells (BGC823) and cervial cancer cells (Hela), Moreover, our recently study showed that $S T Y K 1 / N O K$ protein was also over expressed in gastric cancer and cervical cancer specimens. However, the normal tissues rounding cancer expressed relative lower $S T Y K 1 / N O K$ protein. Interestingly, real-time PCR results showed that the expression of $S T Y K 1 / N O K$ mRNA in tumor cells increased after 2 10 Gy of $\gamma$-ray radiation, which indicated that STYK1/ NOK might participate in the regulation of cancer radio- 
resistance. These results indicated that $S T Y K 1 / N O K$ might be a potential therapeutic target and diagnostic marker for gastric cancer and cervical cancer, and treatment targeting STYK1/NOK might show a synergism with radiotherapy for gastric cancer and cervical cancer. However, there is still a long way to go for the application of STYK1/ NOK, because all the studies on STYK1/ NOK was restricted to laboratory.

\section{Discussion}

The human $S T Y K 1 / N O K$ is widely expressed in human tissues with the highest abundance in the prostate, brain and heart [13]. Besides normal tissues, a variety of tumor cells also expressed the $S T Y K 1 / N O K$ mRNAs [3], such as CRPC (castration-resistant prostate cancer) cells [14]. STYK1/ $N O K$ could promote cell transformation, tumorigenesis, and metastasis [1]. In cells, STYK1/NOK was localised in endosomes and colocalised with epidermal growth factor receptor (EGFR) [15]. STYK1/NOK could mediate STAT3 signaling pathway, RAS/MAPK signaling pathway and PI3K/AKT signaling pathway $[1,16,17]$, and form complexes with both Akt and GSK-3 $\beta$ [2]. Mutation of a tyrosine residue at Y417 site in the catalytic STYK1/NOK domain decreased the tumorigenic potential of tumor cells in vivo, suggesting an oncogenic role for STYK1/NOK [18]. Mutations both Y356F and Y356F significantly impaired Akt phosphorylation and accelerated cell death by activating caspase-3-mediated pathways, but did not affect the kinase activity of $S T Y K 1 / N O K$ [19]. STYK1/ $N O K$ is up-regulated in estrogen receptor-alpha negative estrogen receptor-beta positive breast cancer cells following estrogen treatment [3]. STYK1/NOK is over expressed in ovarian cancer [20], lung cancer [4], breast cancer [19] and acute leukemia [21]. STYK1/NOK mRNA expression did not correlate with c-erbB2 expression, indicating the independence of $S T Y K 1 / N O K$ as a diagnostic marker in breast cancer of tiny tumors in which the malignancy cannot be confirmed by other means [19].

The role study of $S T Y K 1 / N O K$ in gastric cancer and cervical cancer is not reported up to now. Our group is trying to study on this field, and obtained some results. Our preliminary results, together with previous studies, indicate that $S T Y K 1 / N O K$ could be a possible therapeutic target and biomarker for gastric cancer and cervical cancer.

\section{Acknowledgements}

This work was supported by The National Natural Science Foundation of China (fund NO are 81001216 and 81202151), and the Young Scholar Scientific Research Foundation of China CDC (fund NO is 2011A202).

\section{REFERENCES}

[1] L. Liu, X. Z. Yu, T. S. Li, L. X. Song, P. L. Chen, T. L. Suo, Y. H. Li, S. D. Wang, Y. Chen, Y. M. Ren, S. P. Zhang, Z. J. Chang and X. Y. Fu, "A Novel Protein Tyrosine Kinase NOK That Shares Homology with PlateletDerived Growth Factor/Fibroblast Growth Factor Receptors Induces Tumorigenesis and Metastasis in Nude Mice," Cancer Research, Vol. 64, No. 10, 2004, pp. 34913499. doi:10.1158/0008-5472.CAN-03-2106

[2] J. Li, F. Wu, F. Sheng, Y. J. Li, D. Jin, X. Ding and S. Zhang, "NOK/STYK1 Interacts with GSK-3 $\beta$ and Mediates Ser9 Phosphorylation through Activated Akt," FEBS Letters, Vol. 586, No. 21, 2012, pp. 3787-3792. doi:10.1016/j.febslet.2012.09.011

[3] K. S. Kimbro, K. Duschene, M. Willard, J. A. Moore and S. Freeman, “A Novel Gene STYK1/NOK Is Upregulated in Estrogen Receptor-Alpha Negative Estrogen Receptor-Beta Positive Breast Cancer Cells Following Estrogen Treatment," Molecular Biology Reports, Vol. 35, No. 1, 2008, pp. 23-27. doi:10.1007/s11033-006-9047-1

[4] T. Amachika, D. Kobayashi, R. Moriai, N. Tsuji and N. Watanabe, "Diagnostic Relevance of Overexpressed mRNA of Novel Oncogene with Kinase-Domain (NOK) in Lung Cancers," Lung Cancer, Vol. 56, No. 3, 2007, pp. 337340. doi:10.1016/j.lungcan.2007.01.002

[5] D. M. Roder, “The Epidemiology of Gastric Cancer,” Gastric Cancer, Vol. 5 Suppl 1, 2002, pp. 5-11.

[6] B. Zilberstein, C. E. Jacob and I. Cecconello, "Gastric Cancer Trends in Epidemiology," Arquivos de Gastroenterologia, Vol. 49, No. 3, 2012, pp. 177-178. doi:10.1590/S0004-28032012000300001

[7] P. Correa, M. B. Piazuelo and M. C. Camargo, "The Future of Gastric Cancer Prevention,” Gastric Cancer, Vol. 7, No. 1, 2004, pp. 9-16. doi:10.1007/s10120-003-0265-0

[8] T. Nakajima, "Gastric Cancer Treatment Guidelines in Japan,” Gastric Cancer, Vol. 5, No. 1, 2002, pp. 1-5. doi:10.1007/s101200200000

[9] J. Schiedel, A. Blaukat, S. Li, et al., "Matuzumab Binding to EGFR Prevents the Confomational Rearrangement Required for Dimerization," Cancer Cell, Vol. 13, No. 4, 2008, pp. 365-373.

[10] Y. Matsui, M. Inomata, M. Tojigamori, et al., "Suppression of Tumor Growth in Human Gastric Cancer with HER2 Overexpression by an Anli-HER2 Antibody in a Murine Model," International Journal of Oncology, Vol. 27, No. 3, 2005, pp. 68l-685.

[11] K. Fujimoto-Ouchi, F. Sekiguchi, H. Yasuuno, et al., “Antitumor Activity of Trustuzumab in Combination with Chemothempy in Human Gastric Cancer Xenograft Models," Cancer Chemotherapy and Pharmacology, Vol. 59, No. 6, 2007, pp. 795-805.

[12] S. Y. Kim, H. P. Kim, Y. J. Kim, et al., "Traastuzumab Inhibits the Growth of Human Gastric Cancer Cell Lines with HER2 Amplification Synergistically with HER2/ Neu Overexpression/Amplification,” International Journal of Oncology, Vol. 32, No. 1, 2008, pp. 89-95.

[13] X. Ye, C. Ji, Q. Huang, C. Cheng, R. Tang, J. Xu, L. Zeng, J. Dai, Q. Wu, S. Gu, Y. Xie and Y. Mao, "Isola- 
tion and Characterization of a Human Putative Receptor Protein Kinase cDNA STYK1,” Molecular Biology Reports, Vol. 30, No. 2, 2003, pp. 91-96. doi:10.1023/A:1023934017174

[14] S. Chung, K. Tamura, M. Furihata, M. Uemura, Y. Daigo, Y. Nasu, T. Miki, T. Shuin, T. Fujioka, Y. Nakamura and H. Nakagawa, "Overexpression of the Potential Kinase Serine/Threonine/Tyrosine Kinase 1 (STYK 1) in Castrationresistant Prostate Cancer," Cancer Science, Vol. 100, No. 11, 2009, pp. 2109-2114. doi:10.1111/j.1349-7006.2009.01277.X

[15] X. Ding, Q. B. Jiang, R. Li, S. Chen and S. Zhang, "NOK/ STYK1 Has a Strong Tendency towards Forming Aggregates and Colocalises with Epidermal Growth Factor Receptor in Endosomes," Biochemical and Biophysical Research Communications, Vol. 421, No. 3, 2009, pp. 468473. doi:10.1016/j.bbrc.2012.04.016

[16] Y. H. Li, Y. Y. Wang, S. Zhong, Z. L. Rong, Y. M. Ren, Z. Y. Li, S. P. Zhang, Z. J. Chang and L. Liu, "Transmembrane Helix of Novel Oncogene with Kinase-Domain (NOK) Influences Its Oligomerization and Limits the Activation of RAS/MAPK Signaling," Molecules and Cells, Vol. 27, No. 1, 2009, pp. 39-45. doi:10.1007/s10059-009-0003-5

[17] T. Kondoh, D. Kobayashi, N. Tsuji, K. Kuribayashi and N. Watanabe, "Overexpression of Serine Threonine Ty- rosine Kinase 1/Novel Oncogene with Kinase Domain mRNA in Patients with Acute Leukemia,” Experimental Hematology, Vol. 37, No. 7, 2009, pp. 824-830. doi:10.1016/j.exphem.2009.04.010

[18] Y. H. Li, S. Zhong, Z. L. Rong, Y. M. Ren, Z. Y. Li, S. P. Zhang, Z. Chang and L. Liu, "The Carboxyl Terminal Tyrosine 417 Residue of NOK Has an Autoinhibitory Effect on NOK-Mediated Signaling Transductions," Biochemical and Biophysical Research Communications, Vol. 356, No. 2, 2007, pp. 444-449. doi:10.1016/j.bbrc.2007.02.154

[19] R. Moriai, D. Kobayashi, T. Amachika, N. Tsuji and N. Watanabe, "Diagnostic Relevance of Overexpressed NOK mRNA in Breast Cancer,” Anticancer Research, Vol. 26, No. 6C, 2006, pp. 4969-4973.

[20] K. A. Jackson, G. Oprea, J. Handy and K. S. Kimbro, “Aberrant STYK1 Expression in Ovarian Cancer Tissues and Cell Lines,” Journal of Ovarian Research, Vol. 2, No. 1, 2009, pp. 15-22

[21] Y.-H. Li, Y.-Y. Wang, S. Zhong, Z.-L. Rong, Y.-M. Ren, Z.-Y. Li, S.-P. Zhang, Z.-J. Chang and L. Liu, "Transmembrane Helix of Novel Oncogene with Kinase-Domain (NOK) Influences Its Oligomerization and Limits the Activation of RAS/MAPK Signaling," Molecules and Cells, Vol. 27, No. 1, 2009, pp. 39-45. 